\title{
Conserved sex chromosomes and karyotype evolution in monitor lizards (Varanidae)
}

\author{
Alessio lannucci $\mathbb{1}^{1}{ }^{1}$ - Marie Altmanová2,3 ${ }^{2,3}$ Claudio Ciofi ${ }^{1}$ Malcolm Ferguson-Smith ${ }^{4} \cdot$ Massimo Milan ${ }^{5}$. \\ Jorge Claudio Pereira $\mathbb{1}^{4} \cdot$ James Pether ${ }^{6} \cdot$ Ivan Rehák ${ }^{7} \cdot$ Michail Rovatsos $\mathbb{D}^{2,3} \cdot$ Roscoe Stanyon $\mathbb{D}^{1} \cdot$ Petr Velenský ${ }^{7}$. \\ Petr Ráb ${ }^{3} \cdot$ Lukáš Kratochvíl (i) ${ }^{2} \cdot$ Martina Johnson Pokorná2,3
}

Received: 15 September 2018 / Revised: 10 December 2018 / Accepted: 14 December 2018 / Published online: 22 January 2019

(c) The Genetics Society 2019

\begin{abstract}
Despite their long history with the basal split dating back to the Eocene, all species of monitor lizards (family Varanidae) studied so far share the same chromosome number of $2 n=40$. However, there are differences in the morphology of the macrochromosome pairs 5-8. Further, sex determination, which revealed ZZ/ZW sex microchromosomes, was studied only in a few varanid species and only with techniques that did not test their homology. The aim of this study was to (i) test if cryptic interchromosomal rearrangements of larger chromosomal blocks occurred during the karyotype evolution of this group, (ii) contribute to the reconstruction of the varanid ancestral karyotype, and (iii) test homology of sex chromosomes among varanids. We investigated these issues by hybridizing flow sorted chromosome paints from Varanus komodoensis to metaphases of nine species of monitor lizards. The results show that differences in the morphology of the chromosome pairs 5-8 can be attributed to intrachromosomal rearrangements, which led to transitions between acrocentric and metacentric chromosomes in both directions. We also documented the first case of spontaneous triploidy among varanids in Varanus albigularis. The triploid individual was fully grown, which demonstrates that polyploidization is compatible with life in this lineage. We found that the $\mathrm{W}$ chromosome differs between species in size and heterochromatin content. The varanid $\mathrm{Z}$ chromosome is clearly conserved in all the analyzed species. Varanids, in addition to iguanas, caenophidian snakes, and lacertid lizards, are another squamate group with highly conserved sex chromosomes over a long evolutionary time.
\end{abstract}

\section{Introduction}

Varanids, also known as monitor lizards, are squamate reptiles belonging to the suborder Anguimorpha, which

These authors contributed equally to this work: Alessio Iannucci, Marie Altmanová

Supplementary information The online version of this article (https:// doi.org/10.1038/s41437-018-0179-6) contains supplementary material, which is available to authorized users.

Lukáš Kratochvíl

lukas.kratochvil@natur.cuni.cz

1 Department of Biology, University of Florence, Via Madonna del Piano 6, 50019 Sesto Fiorentino, FI, Italy

2 Department of Ecology, Charles University, Viničná 7, 12800 Prague, Czech Republic

3 Institute of Animal Physiology and Genetics, The Czech Academy of Sciences, Rumburská 89, 27721 Liběchov, Czech Republic together with iguanians and snakes forms the clade Toxicofera (Vidal and Hedges 2005). The family Varanidae comprises a single extant genus, Varanus, which split from the sister group, the family Lanthanotidae, 70 MYA (Lin and Wiens 2017). Currently, 80 extant species of varanids are described (Uetz and Hošek 2017) and karyotypes have been reported for 24 Varanus species (Table 1). All these species have a diploid chromosome number of $2 n=40$, consisting of 16 macrochromosomes and 24 microchromosomes. This consistency in chromosome number

4 Cambridge Resource Centre for Comparative Genomics, Department of Veterinary Medicine, University of Cambridge, Madingley Road, Cambridge CB3 OES, United Kingdom

5 Department of Comparative Biomedicine and Food Science, University of Padova, Viale dell'Università 16, 35020 Legnaro, PD, Italy

6 Reptilandia Park, Galdar 35460 Gran Canaria, Spain

7 Prague Zoological Garden, U Trojského zámku 120/3, 17100 Prague, Czech Republic 
Table 1 Basic cytogenetic data in Varanidae

\begin{tabular}{|c|c|c|c|c|c|c|}
\hline Clade & Species & Subspecies & Reference & $2 n$ & Karyotype & Sex chromosomes \\
\hline \multirow[t]{9}{*}{ niloticus clade } & \multirow[t]{2}{*}{ V. albigularis } & & King and King $(1975)^{* 1}$ & 40 & 40: $16(8,8) 24$ & $\mathrm{ZW}$ \\
\hline & & albigularis & Present study & $3 n=60^{\ddagger}$ & 60: $24(12,12) 36$ & \\
\hline & \multirow[t]{3}{*}{ V. exanthematicus } & & De Smet $(1981)^{\dagger}$ & 40 & 40: $16(8,8) 24$ & \\
\hline & & & Srikulnath et al. (2013) & 40 & 40: $16(10,6) 24$ & \\
\hline & & & Present study & 40 & 40: $16(10,6) 24$ & \\
\hline & \multirow[t]{2}{*}{$V$. griseus } & griseus & King and King (1975) & 40 & 40: $16(16,0) 24$ & \\
\hline & & & De Smet $(1981)^{\dagger}$ & 40 & 40: $16(14,2) 24$ & \\
\hline & \multirow[t]{2}{*}{ V. niloticus } & niloticus & King and King (1975) & 40 & 40: $16(8,8) 24$ & $\mathrm{ZW}$ \\
\hline & & & Porter et al. (1994) & 40 & 40: $16(8,8) 24$ & \\
\hline \multirow[t]{5}{*}{ indicus clade } & V. indicus & indicus & King and King (1975) & 40 & 40: $16(14,2) 24$ & \\
\hline & V. beccarii & & Present study & 40 & 40: $16(14,2) 24$ & ZW \\
\hline & V. boehmei & & Present study & 40 & 40: $16(14,2) 24$ & \\
\hline & V. macraei & & Present study & 40 & 40: $16(14,2) 24$ & ZW \\
\hline & $V \cdot$ prasinus & & Present study & 40 & 40: $16(14,2) 24$ & ZW \\
\hline \multirow[t]{12}{*}{ salvator clade } & \multirow[t]{4}{*}{$V$. bengalensis } & & Dutt $(1968)^{\dagger}$ & 40 & 40: $16(8,8) 24$ & \\
\hline & & bengalensis & Singh et al. (1970), Singh (1974) & 40 & 40: $16(14,2) 24$ & \\
\hline & & & De Smet $(1981)^{\dagger}$ & 40 & 40: $16(16,0) 24$ & \\
\hline & & & Patawang et al. (2017a, 2017b) & 40 & 40: $16(14,2) 24$ & ZW \\
\hline & $V$. flavescens & & Singh et al. (1970), Singh (1974) & 40 & $40: 16(14,2) 24$ & \\
\hline & V. nebulosus & & King and King $(1975)^{* 2}$ & 40 & 40: $16(14,2) 24$ & \\
\hline & \multirow[t]{2}{*}{ V. rudicollis } & & Gorman and Gress (1970) & 40 & 40: $16(14,2) 24$ & \\
\hline & & & Present study & 40 & 40: $16(14,2) 24$ & \\
\hline & \multirow[t]{4}{*}{ V. salvator } & salvator & King and King (1975) & 40 & 40: $16(14,2) 24$ & \\
\hline & & & De Smet $(1981)^{\dagger}$ & 40 & 40: $16(16,0) 24$ & \\
\hline & & macromaculatus & Srikulnath et al. (2013) & 40 & 40: $16(14,2) 24$ & \\
\hline & & & Present study & 40 & 40: $16(14,2) 24$ & $\mathrm{ZW}$ \\
\hline \multirow[t]{2}{*}{ varius clade } & V. varius & & King and King (1975) & 40 & 40: $16(14,2) 24$ & ZW \\
\hline & V. komodoensis & & Johnson Pokorná et al. (2016) & 40 & 40: $16(14,2) 24$ & ZW \\
\hline \multirow[t]{11}{*}{ gouldii clade } & V. giganteus & & King and King (1975) & 40 & 40: $16(10,6) 24$ & \\
\hline & \multirow[t]{4}{*}{ V. gouldii } & & Matthey $(1931)^{\dagger}$ & 40 & 40: $16(8,8) 24$ & \\
\hline & & gouldii & King and King (1975) & 40 & 40: $16(10,6) 24$ & \\
\hline & & flavirufus & King and King (1975) & 40 & 40: $16(10,6) 24$ & \\
\hline & & & Matsubara et al. (2014) & 40 & 40: $16(10,6) 24$ & ZW \\
\hline & \multirow[t]{2}{*}{$V$. mertensi } & & King and King (1975) & 40 & 40: $16(10,6) 24$ & \\
\hline & & & Present study & 40 & 40: $16(10,6) 24$ & ZW \\
\hline & $V \cdot$ panoptes & horni & Present study & 40 & 40: $16(10,6) 24$ & \\
\hline & \multirow[t]{2}{*}{$V$. rosenbergi } & & King and King $(1975)^{* 3}$ & 40 & 40: $16(10,6) 24$ & \\
\hline & & & Matsubara et al. (2014) & 40 & 40: $16(10,6) 24$ & ZW \\
\hline & V. spenceri & & King and King (1975) & 40 & 40: $16(10,6) 24$ & \\
\hline \multirow[t]{5}{*}{ tristis clade } & V. glauerti & & Present study & 40 & 40: $16(14,2) 24$ & ZW \\
\hline & V. scalaris & & King and King (1975) & 40 & 40: $16(14,2) 24$ & \\
\hline & $V$. semiremex & & King and King (1975) & 40 & 40: $16(14,2) 24$ & \\
\hline & V. timorensis & similis & King and King (1975) & 40 & 40: $16(14,2) 24$ & \\
\hline & V. tristis & orientalis & King and King (1975) & 40 & 40: $16(14,2) 24$ & \\
\hline \multirow[t]{3}{*}{ acanthurus clade } & \multirow[t]{3}{*}{ V. acanthurus } & & King et al. (1982) & 40 & 40: $16(14,2) 24$ & ZW \\
\hline & & & Matsubara et al. (2014) & 40 & 40: $16(14,2) 24$ & ZW \\
\hline & & & Present study & 40 & 40: $16(14,2) 24$ & $\mathrm{ZW}$ \\
\hline
\end{tabular}


Table 1 (continued)

\begin{tabular}{llllll}
\hline Clade & Species & Subspecies & Reference & $2 n$ & Karyotype \\
\hline V. gilleni & & King and King (1975) & 40 & $40: 16(14,2) 24$ \\
& V. storri & King and King (1975) & 40 & $40: 16(14,2) 24$ \\
\hline
\end{tabular}

Karyotypes are described as follows: diploid chromosome number: number of macrochromosomes (number of bi-armed macrochromosomes, number of uniarmed macrochromosomes) number of microchromosomes

${ }^{* 1}$ Referred as V. exanthematicus albigularis therein

${ }^{* 2}$ Referred as $V$. bengalensis nebulosus therein

${ }^{* 3}$ Referred as $V$. gouldii rosenbergi therein

$\dagger$ Karyotypes described in these references were not considered in the ancestral state reconstruction

$\ddagger$ Likely a case of spontaneous triploidy

and type suggests that the genome organisation might be highly conserved across the whole family. However, the morphology of certain chromosomes shows some variability among varanid species. As microchromosomes are hardly distinguishable by morphology, they were not included in these comparisons. The largest two pairs of macrochromosomes are metacentric and the pairs 3 and 4 are medium-sized bi-armed chromosomes in all species studied so far. Some variability exists in the morphology of the macrochromosomal pairs 5-8. In most species (Table 1), pair 5 is acrocentric and pairs 6,7 , and 8 are bi-armed chromosomes. Although this karyotype was considered to be ancestral for varanids (King and King 1975; Chaiprasertsri et al. 2013; Srikulnath et al. 2013), as yet there has been no robust phylogenetic reconstruction of the ancestral morphology of the varanid macrochromosomes.

Squamate reptiles do have, however, well-known variability in sex determination systems, from environmental to genotypic sex determination. Genotypic sex determination involves sex chromosomes at various stage of differentiation, comprising male $(\mathrm{XX} / \mathrm{XY})$ or female $(\mathrm{ZZ} / \mathrm{ZW})$ heterogamety (Valenzuela et al. 2003; Janzen and Krenz 2004; Sarre et al. 2004; Valenzuela and Lance 2004; Pokorná and Kratochvíl 2009; Johnson Pokorná and Kratochvíl 2016). Among varanids, sex chromosomes have been reported for only eight species (Table 1). All these species have female heterogamety with differentiated ZZ/ZW sex chromosomes, where the $\mathrm{W}$ is distinguishable by its highly heterochromatic region. In some species the $\mathrm{W}$ chromosome is distinctively larger than the other microchromosomes (King and King 1975; Matsubara et al. 2014). Differences in accumulations of microsatellite motifs on the W chromosome were reported among three species studied by Matsubara et al. (2014). Chromosome $\mathrm{Z}$ was not yet identified in any varanid species and data on homology of sex chromosomes among varanids are lacking.

Recently, Iannucci et al. (2019) produced a set of chromosome painting probes from a female $V$. komodoensis by flow sorting. In this study, we hybridized this set of probes
Table 2 List of individuals analyzed

\begin{tabular}{|c|c|c|}
\hline Clade & Species & $\begin{array}{l}\text { Number and sex of } \\
\text { individuals }\end{array}$ \\
\hline \multirow[t]{2}{*}{ niloticus } & V. albigularis albigularis & 1unknown sex \\
\hline & V. exanthematicus & 10 \\
\hline \multirow[t]{4}{*}{ indicus } & V. beccarii & 10,19 \\
\hline & V. boehmei & $10^{\star}$ \\
\hline & V. macraei & 10,19 \\
\hline & $V \cdot$ prasinus & 10,19 \\
\hline \multirow[t]{2}{*}{ salvator } & V. rudicollis & $10^{\star}$ \\
\hline & V. salvator & $10,2 \%$ \\
\hline \multirow[t]{2}{*}{ gouldii } & V. mertensi & 10,19 \\
\hline & $V \cdot$ panoptes horni & $10^{\top}$ \\
\hline tristis & V. glauerti & 10,19 \\
\hline acanthurus & V. acanthurus & 10,49 \\
\hline
\end{tabular}

to a number of varanid species to (i) test if cryptic interchromosomal rearrangements of larger chromosomal blocks occurred during the karyotype evolution of this group, (ii) contribute to the reconstruction of the varanid ancestral karyotype, and (iii) test homology of sex chromosomes among varanids.

\section{Material and methods}

\section{Studied species}

The molecular phylogenetic study by Vidal et al. (2012) split the genus Varanus into seven distinct clades: niloticus, indicus, salvator, varius, gouldii, tristis, and acanthurus. This division is generally well supported also by more recent phylogenetic analyses (Zheng and Wiens 2016; Lin and Wiens 2017). We worked on 12 species of monitor lizards with a key phylogenetic position, representing all seven major varanid clades (Table 2). Males and females 
were analyzed in seven of these species, whereas only males in the remaining species. The sex of the individuals was identified based on breeding history, sexual behavior, or morphology. Blood samples were obtained from animals originating from the pet trade, private breeders or Czech zoological gardens (Zoo Praha, Zoo Plzeň) under the supervision and with the approval of the Ethics Committee of the Faculty of Science, Charles University in Prague followed by the Committee for Animal Welfare of the Ministry of Agriculture of the Czech Republic (permission No. 35484/2015-14).

\section{Chromosomal preparations, staining, and karyotyping}

Metaphase chromosome spreads were prepared from whole blood cell cultures, following the protocol described in Pokorná et al. (2010) with slight modifications. Chromosomal preparations were stained with conventional Giemsa solution. C-banding staining was performed following Sumner (1972) as described in Pokorná et al. (2014). Chromosomes were arranged according to their size. When results from chromosome painting were available, karyotypes of the analyzed species were arranged based on homology with the karyotype of $V$. komodoensis (Iannucci et al. 2019). Karyotypes were arranged using the software Ikaros (MetaSystems).

\section{Chromosome-specific probes}

Preparation and labeling of $V$. komodoensis (VKO) chromosome-specific painting probes are described in detail in Iannucci et al. (2019). Briefly, chromosomes obtained by fibroblast cultivation were sorted using a Mo-Flo ${ }^{\circledR}$ (Beckman Coulter) cell sorter. Genetic material was then amplified and labeled by degenerate oligonucleotide primed PCR. Each $V$. komodoensis paint for macrochromosomes is specific for one single pair of chromosomes, except for $\mathrm{VKO}$ / 7 and $\mathrm{VKO} / 7$, which contain one specific chromosome pair each (pair 6 and pair 8, respectively), plus a third pair that overlaps between the two of them (pair 7). The $\mathrm{W}$ and $\mathrm{Z}$ chromosomes are contained in probes VKO11/12/W and VKO17/18/Z, respectively, together with two pairs of other microchromosomes each (Fig. 1).

\section{Cross-species chromosome painting}

V. komodoensis paints for macrochromosomes and sex chromosomes were hybridized onto chromosomes of male individuals of $V$. exanthematicus, $V$. prasinus, $V$. rudicollis, V. salvator, V. mertensi, V. panoptes horni, V. glauerti, and $V$. acanthurus and of individual of unknown sex of $V$. albigularis albigularis. VKO11/12/W and
VKO17/18/Z were hybridized also to female individuals of $V$. prasinus, V. salvator, V. mertensi, V. glauerti, and $V$. acanthurus. Fluorescence in situ hybridization (FISH) experiments were performed as described in Iannucci et al. (2019). Images were captured using a Provis AX70 (Olympus) fluorescence microscope equipped with a DP30BW digital camera (Olympus). DP manager imaging software (Olympus) was used to capture gray scale images and to superimpose the source images with colors to visualize the results of FISH.

\section{Ancestral state reconstruction}

The phylogeny of cytogenetic characters, i.e., differences in the morphology of chromosome pairs 5-8, among varanids was reconstructed by maximum parsimony (Fitch 1971; Dobigny et al. 2004) using Mesquite v.3.51 (Maddison and Maddison 2018). The analyses were based on the phylogenetic tree of Lin and Wiens (2017). However, we took into account also the alternative topology of $V$. griseus, which is sister to other species of the niloticus clade in Lin and Wiens (2017), but sister to all species that do not belong to the niloticus clade in Zheng and Wiens (2016). In species where conflicting data were available, we included only the best data with respect to species identification, description of sampling locality, number of individuals studied, and quality of karyotype pictures (Table 1).

\section{Results}

\section{Karyotypes and sex chromosomes}

Karyotypes of $V$. beccarii, $V$. boehmei, $V$. macraei, $V$. prasinus, V. panoptes horni, and $V$. glauerti were described for the first time in this study. The cytogenetic analyses revealed that the 11 species analyzed all have a diploid number of $2 n=40$, consisting of 8 pairs of macrochromosomes and 12 pairs of microchromosomes (Fig. 2). The only exception is $V$. albigularis albigularis, which was triploid with $3 n=60$ chromosomes (Fig. 2a). Chromosome pairs (triplets in the case of $V$. albigularis albigularis) 1, 2, 3, and 4 have the same morphology in all species. Pairs 1 and 2 are large metacentric, whereas pairs 3 and 4 are medium-sized (sub)metacentric chromosomes. Pair 5 is acrocentric in all species except $V$. exanthematicus, where it is submetacentric. Pairs 6, 7, and 8 are (sub)metacentric in $V$. beccarii, V. boehmei, V. macraei, V. prasinus, V. rudicollis, V. salvator, V. glauerti, and V. acanthurus. Pairs 6 and 7 are acrocentric, whereas pair 8 is metacentric in $V$. mertensi and $V$. panoptes horni. $V$. exanthematicus has pairs 6,7 , and 8 acrocentric. $V$. albigularis albigularis triplets 6,7 , and 8 are acrocentric as well. The morphology of the 


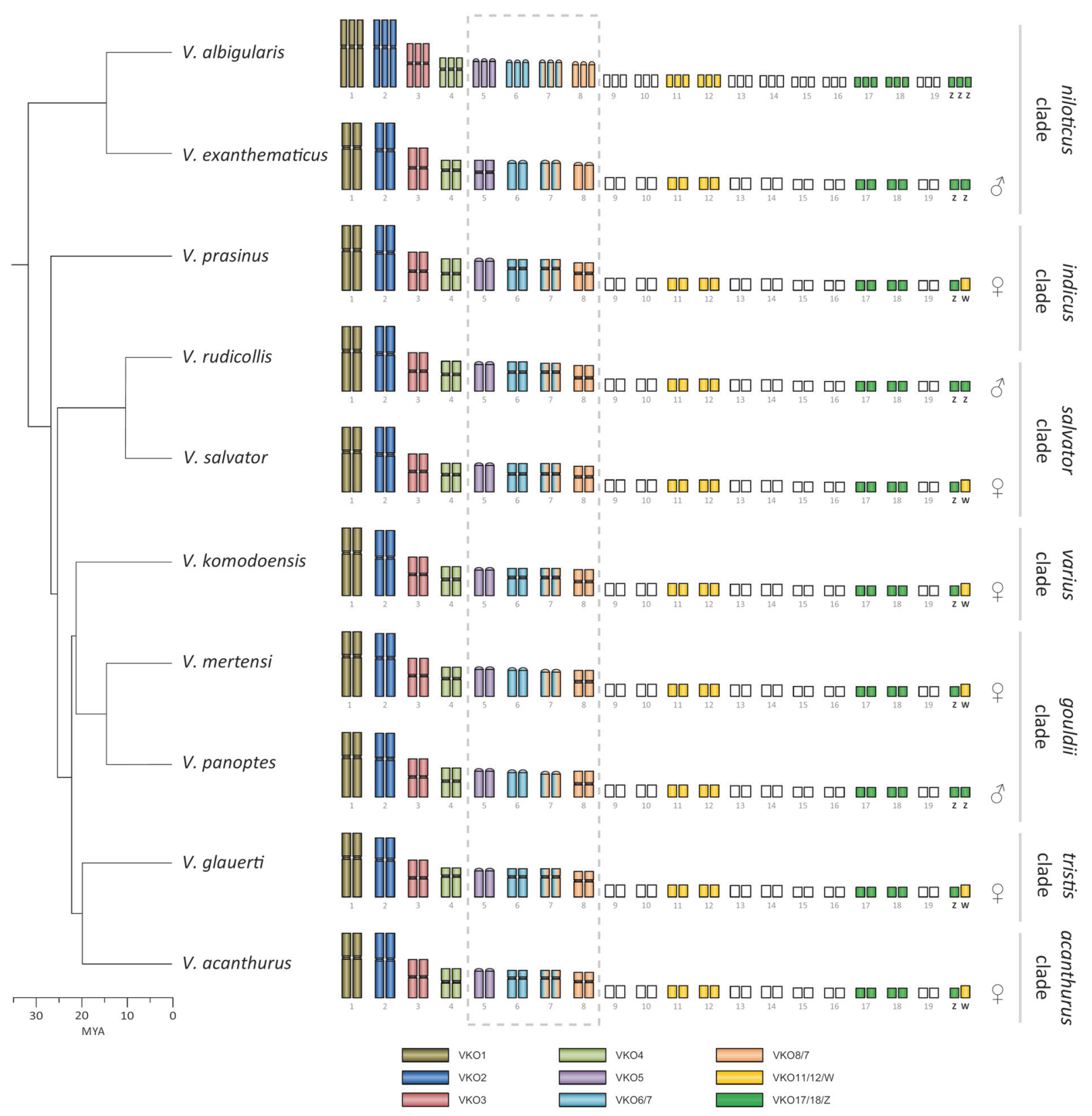

Fig. 1 Schematic representation of the results of the FISH experiments with Varanus komodoensis chromosome paints. Results of female individuals are shown only for those species in which both sexes were

microchromosomes is not identifiable because of their small sizes (Fig. 2).

C-banding revealed variable heterochromatic patterns in the pericentromeric and telomeric regions of several macrochromosome and microchromosome pairs (Fig. 2). A strongly heterochromatic microchromosome was detected via C-banding in females of $V$. beccarii, V. macraei, $V$. prasinus, V. mertensi, V. glauerti, and $V$. acanthurus. This heterochromatic chromosome was absent in male metaphases, suggesting that it could represent the $\mathrm{W}$ chromosome (Fig. 2). C-banded karyotypes were similar in both studied. Phylogenetic relationships follow Lin and Wiens (2017). In $V$. albigularis albigularis, the studied individual was likely an exceptional spontaneous triploid

sexes of $V$. salvator (Fig. 2k, 1). The larger unpaired microchromosome in male and female karyotype in $V$. acanthurus reported by Matsubara et al. (2014) and interpreted there as a likely polymorphism of an autosomal microchromosome was not observed in our study.

\section{Chromosome painting}

Chromosome painting demonstrates a strong conservation of macrochromosomes (Fig. 1; Suppl. Material 1). $V$. komodoensis macrochromosome pairs 1, 2, 3, and 4 are 

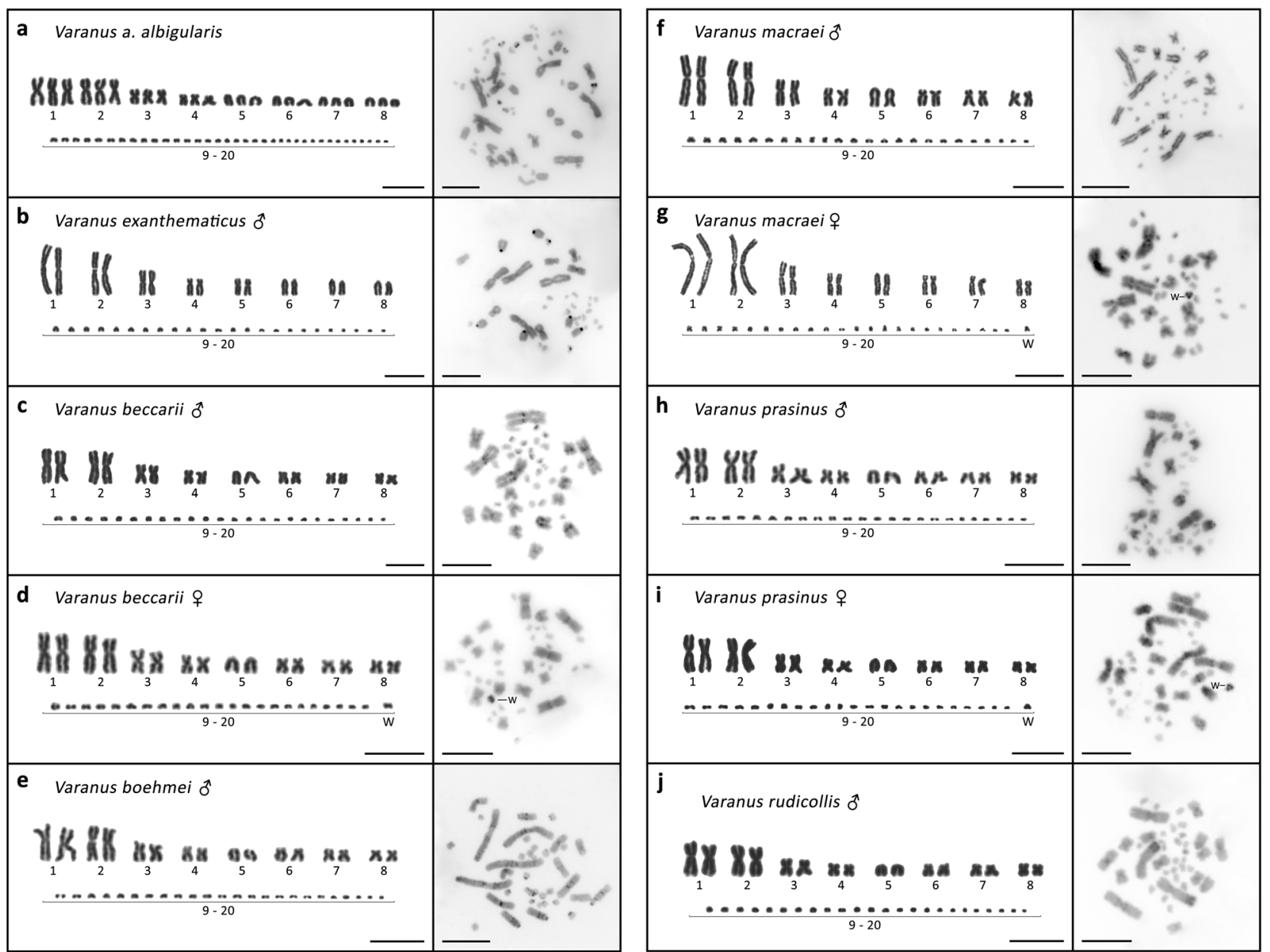

Fig. 2 Giemsa stained karyotype (left) and C-banded metaphases (right) of analyzed species. The W chromosomes in female karyotype were identified by sequential C-banding. The $\mathrm{Z}$ chromosomes are not assigned due to their similarity to other microchromosomes. Scale bars $=10 \mu \mathrm{m}$

conserved in both morphology and painting pattern in other monitor lizards (e.g., Fig. 3a-c). Painting with VKO5, $\mathrm{VKO} / 7$, and VKO8/7 gave the same results obtained for $V$. komodoensis, i.e., VKO5 painted chromosome 5, whereas VKO6/7 and VKO8/7 painted chromosomes 6 and 8, respectively, and pair 7 , confirming the conservation in synteny for these four pairs of chromosomes despite their morphological diversity (e.g., Fig. 3d-f). Triploidy of $V$. albigularis was confirmed as all the paints painted triplets instead of pairs of chromosomes (e.g., Fig. 3g, h).

Hybridization of VKO11/12/W and VKO17/18/Z to male individuals resulted in painting four and six microchromosomes, respectively (e.g., Fig. 3i, k). VKO11/12/W hybridized to female metaphases painted five microchromosomes including the W (e.g., Fig. 3j). VKO17/18/Z probe painted five microchromosomes, one of which is probably the $\mathrm{Z}$ (e.g., Fig. 31). Identical results were obtained for $V$. komodoensis by Iannucci et al. (2019). A comparison of hybridization pattern of VKO11/12/W and VKO17/18/Z in the same individual revealed that $\mathrm{W}$ chromosome is usually larger than $\mathrm{Z}$ chromosome in varanids (e.g., Fig. $3 i-1)$.

\section{Ancestral state reconstruction}

The ancestral state reconstruction did not unequivocally identify the ancestral pattern of the morphology for chromosomes 5-8 in varanids (Fig. 4). The ancestral state of chromosome pair 5, could be either submetacentric as in $V$. griseus or acrocentric. In any case, the situation in $V$. exanthematicus seems to be an apomorphy of this species. Our analysis suggests that chromosome pair 5 was subject to rearrangement from an acrocentric to a metacentric shape in the ancestor of $V$. exanthematicus. The ancestral state of chromosomes 6-8 for varanids could be submetacentric as in $V$. griseus, acrocentric, or metacentric. It appears that chromosomal rearrangements transformed chromosome pair 6 and 7 from the ancestral metacentrics in the common ancestor of the gouldii group to acrocentrics. The alternative topology of $V$. griseus suggested by Zheng and Wiens 


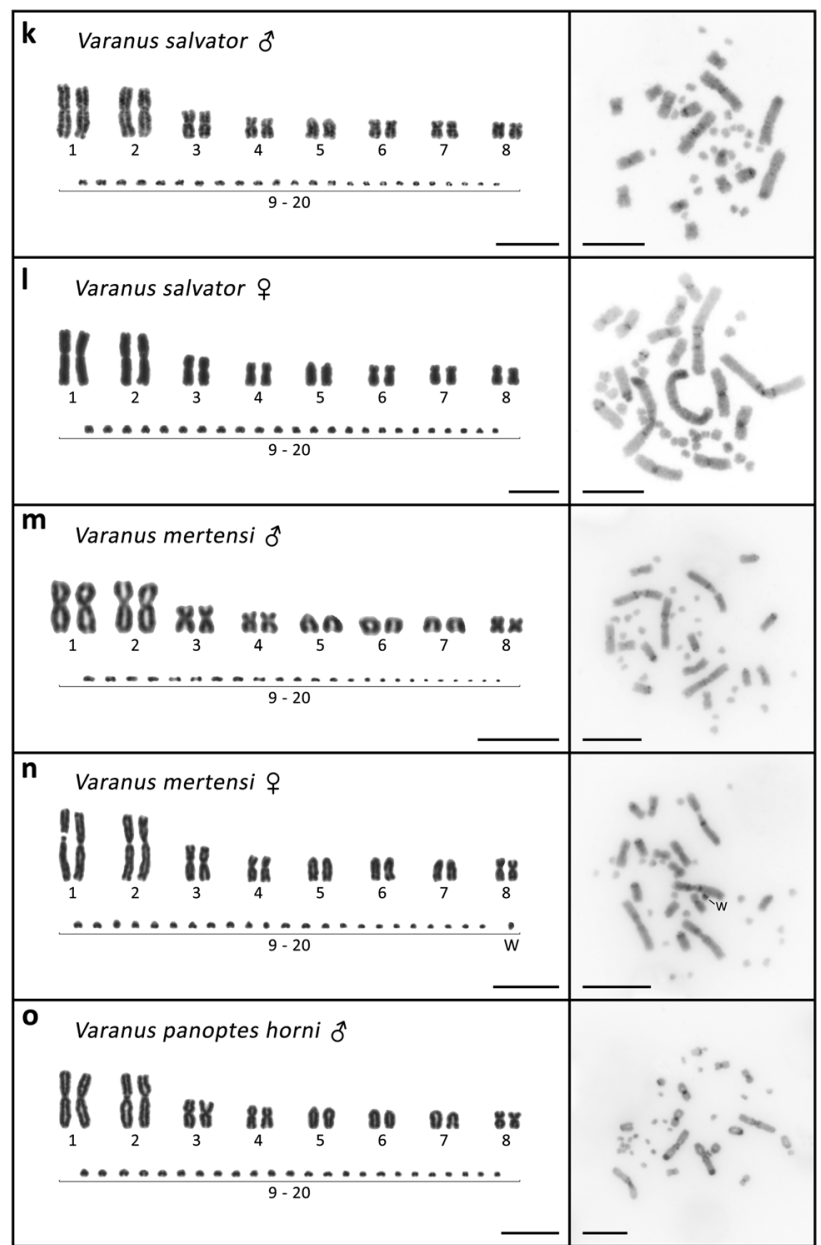

Fig. 2 (continued)

(2016) causes only a single change in interpretation of the phylogenetic pattern. In this case, the acrocentric shape of chromosome 5 is considered as ancestral for all varanids and the submetacentric chromosome of $V$. griseus is an apomorphy of this species.

\section{Discussion}

\section{Karyotype evolution in monitor lizards}

Our results strongly suggest that the chromosome number of $2 n=40$ is ancestral for varanids (Fig. 4). However, the lack of cytogenetic information on the accepted closest varanid outgroup, the sister family Lanthanotidae (Pyron et al. 2013; Zheng and Wiens 2016; Lin and Wiens 2017), prevents us from arriving at final conclusions on the composition of the varanid ancestral karyotype. Recently, the karyotype of the Gila monster, Heloderma suspectum, was described by Johnson Pokorná et al. (2014). The karyotype of this anguimorphan species has $2 n=36$ chromosomes (14

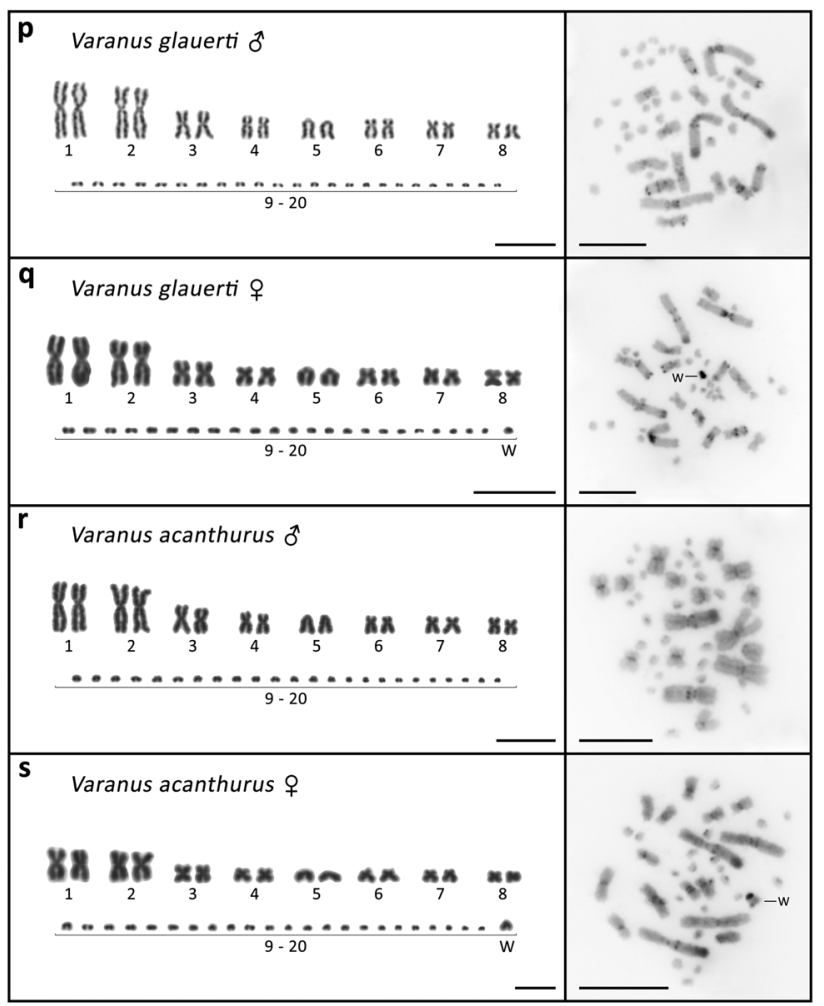

macro- and 22 microchromosomes) and it is similar to karyotypes found in more distant varanid outgroups such as Iguania and snakes (Singh 1972; Altmanová et al. 2016; Olmo and Signorino 2016). Some rearrangements must have occurred along the evolutionary pathway from the last common ancestor of the varanids and the Gila monster, most likely in the lineage leading to the varanids. These rearrangements probably involved microchromosomes (Srikulnath et al. 2013).

The situation concerning the morphology of macrochromosomes 5-8 in the ancestral varanid karyotype is more complicated. In their pioneering work, King and King (1975) assumed that during evolution pericentric inversions tended to change these chromosomes from predominantly bi-armed to acrocentric. Therefore, they viewed the karyotype of the salvator clade as ancestral for varanids. However, this hypothesis does not correspond to the conclusions from current phylogenetic reconstructions (Pianka and King 2004; Lin and Wiens 2017). The results of the ancestral state reconstruction show that the ancestral situation for all varanids is not yet resolved (Fig. 4). It largely 


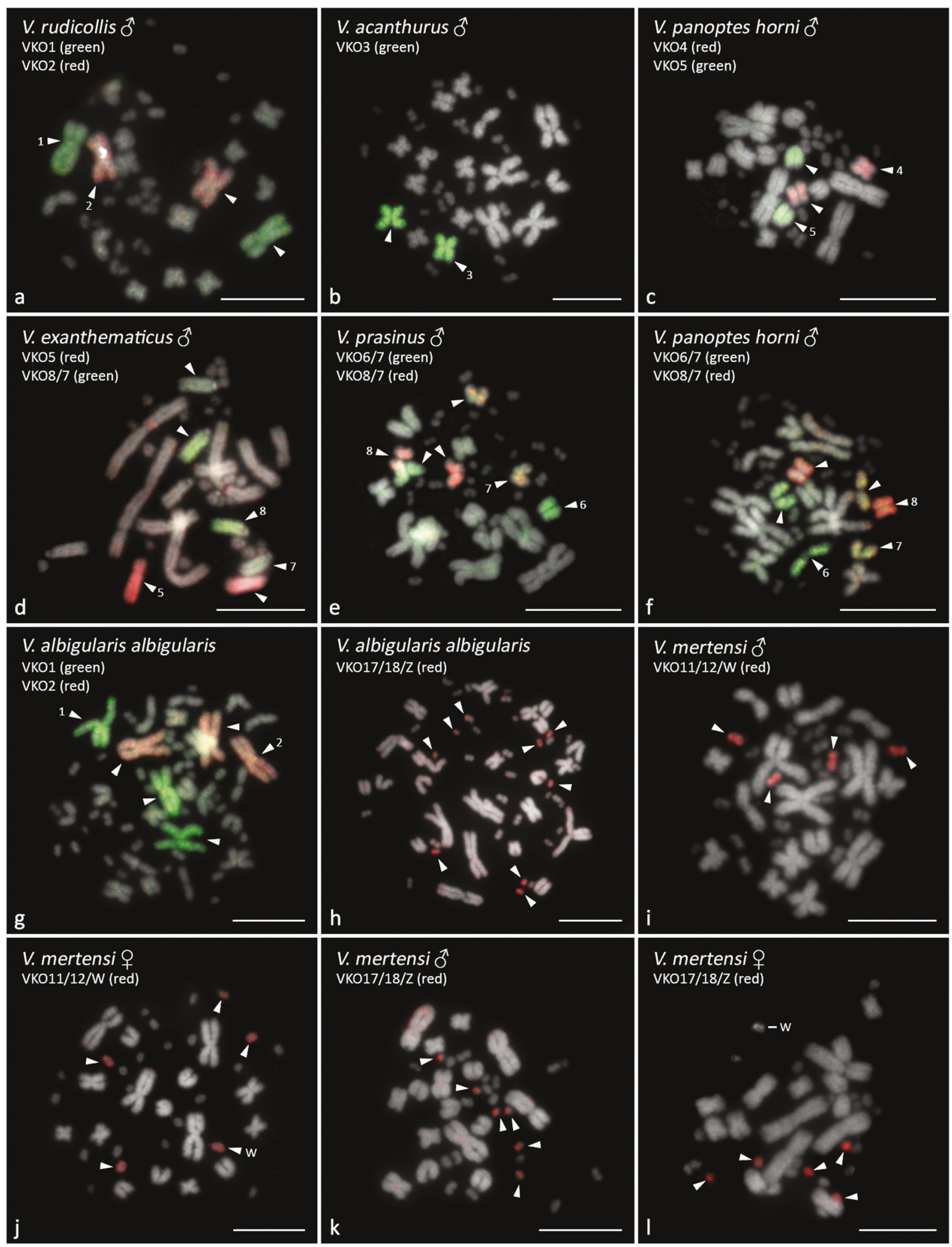

Fig. 3 Fluorescence in situ hybridization of Varanus komodoensis chromosomes paints. Arrowheads point to hybridization signals. Numbers are not given to microchromosomes as pairs are not distinguishable from each other by morphology. Scale bars $=10 \mu \mathrm{m}$ 


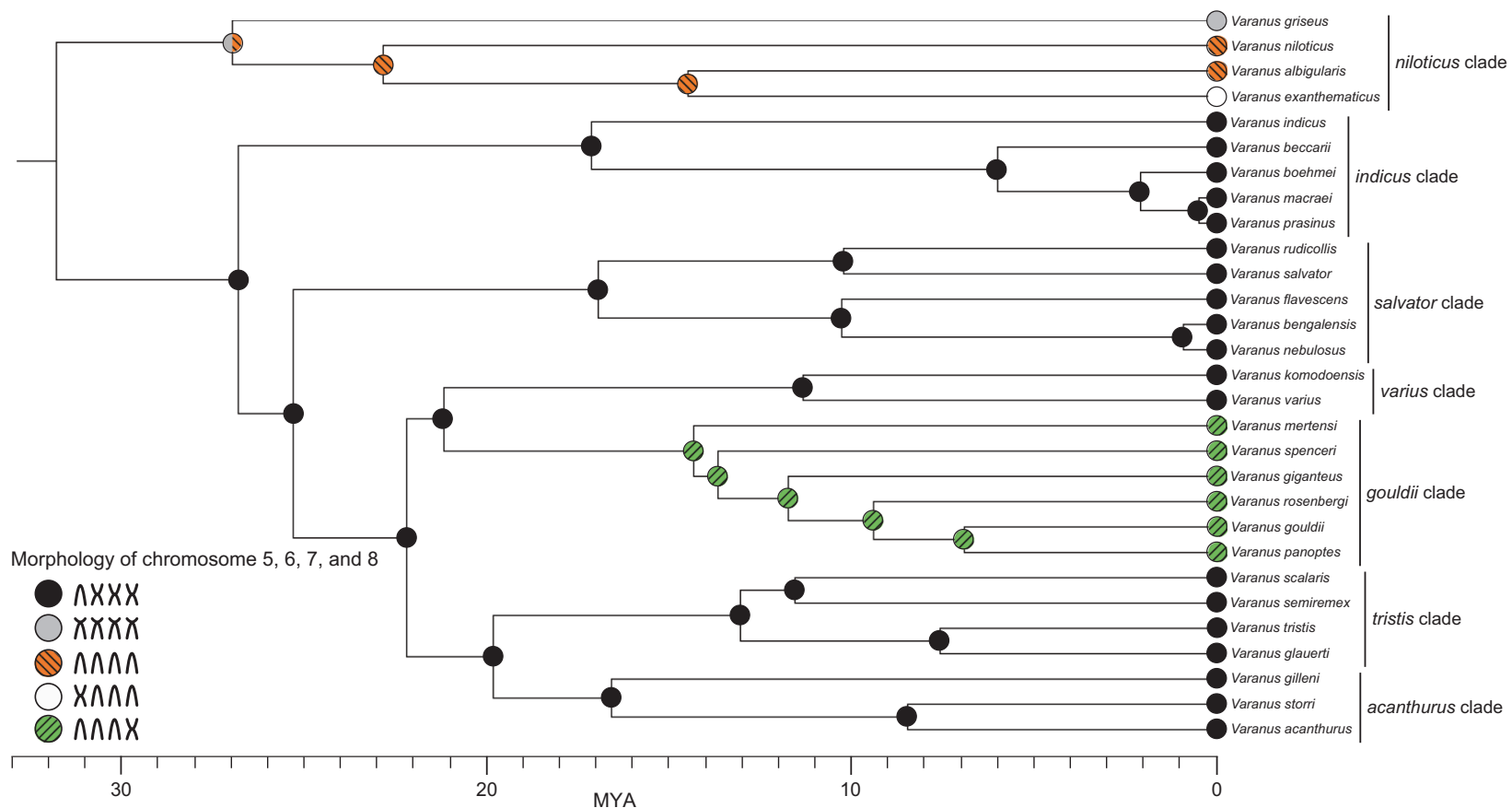

Fig. 4 Ancestral state reconstruction of morphology of chromosome pairs 5-8 using maximum parsimony. The phylogenetic relationships follow Lin and Wiens (2017)

depends on the homology of the morphology of chromosome pairs 5-8 between $V$. griseus and the majority of varanid species. Chromosome pairs 5-8 in V. griseus were considered to be submetacentric with remarkably shorter parms or even "subacrocentric" (King and King 1975), whereas chromosomes 6-8 are metacentric and the chromosome pair 5 is acrocentric in most of the other varanids. One hypothesis is that the morphology of chromosomes 5-8 may be apomorphic in V. griseus. However, a detailed comparison of gene order in $V$. griseus and other varanids might be needed to test this hypothesis. Regardless of the ancestral morphology of chromosomes 5-8, the patterns of chromosome morphology observed across species suggest that changes in macrochromosomes from acrocentrics to metacentrics went in both directions (Fig. 4). Chromosome painting data show that these changes between metacentric and acrocentric chromosomes can be attributed solely to intrachromosomal rearrangements involving pericentromeric inversion or centromere repositioning, and not to interchromosomal translocations.

Our results using chromosome painting do not support some of the conclusions from the study by Srikulnath et al. (2013) based on physical mapping of 17 functional genes in $V$. salvator and $V$. exanthematicus. These authors suggested that acrocentric chromosome pairs 6 and 8 of $V$. exanthematicus were homologous to two metacentric pairs of $V$. salvator and that a metacentric pair assigned as pair 7 in Srikulnath et al. (2013) of $V$. exanthematicus was homologous to the submetacentric pair 7 of V. salvator. Instead, our chromosome painting results showed that metacentric chromosome pairs 6,7 , and 8 of $V$. salvator are homologous to three acrocentric pairs of $V$. exanthematicus. Further, the acrocentric chromosome pair 5 of $V$. salvator is homologous to a submetacentric pair of $V$. exanthematicus. These results suggest that the acrocentric morphology of chromosome pairs 6,7 , and 8 is a conserved feature in the niloticus clade (Fig. 1).

In summary, unlike mammals, where high rates of interchromosomal rearrangements were found (FergusonSmith and Trifonov 2007), chromosome painting data on varanids strongly support that similar to birds, squamate reptiles have a rather high rate of intrachromosomal and a low rate of interchromosomal rearrangements. This characteristic is probably common for the whole sauropsid group (Alföldi et al. 2011; Pokorná et al. 2011a, 2012; Skinner and Griffin 2012; Lithgow et al. 2014; Rovatsos et al. 2014a; Johnson Pokorná et al. 2015). The same karyotypic stability found in varanids was also described for other squamate lineages such as oplurids (Altmanová et al. 2016), skinks (Giovannotti et al. 2010) and geckos (Shibaike et al. 2009; Trifonov et al. 2011; Johnson Pokorná et al. 2015), even if Robertsonian rearrangements have been documented in some of these groups (Pokorná et al. 2010). On the other hand, a high karyotypic variability has been registered for chameleons (Rovatsos et al. 2017). A broader study involving comparative mapping or genome sequencing of representative species of these groups is necessary to determine whether any cryptic inter- or intrachromosomal 
rearrangements occurred in the lineages with conserved karyotypes. It is not yet clear why some squamate lineages have more variable karyotypes than others, which is a more important and general question.

\section{Sex chromosome evolution}

Hybridization of probes containing $\mathrm{Z}$ chromosome of $\mathrm{V}$. komodoensis onto metaphases of male and female individuals in other species revealed that the $\mathrm{Z}$ chromosome is conserved among all the species tested, supporting strong homology of sex chromosomes among the varanid clades indicus, salvator, gouldii, tristis, and acanthurus (Suppl. Material 1). Thus, the origin of the sex chromosomes in monitor lizards can be dated back at least to 27-31 MYA, which is the estimated age of the split between the indicus clade and the group containing the other mentioned varanid clades (Zheng and Wiens 2016; Lin and Wiens 2017). We did not have any female of a species from the niloticus clade available for a direct test for sex chromosome homology, however, earlier detection of ZZ/ZW sex chromosomes in species from this clade (Table 1) suggests that varanid sex chromosomes can be even older than the basal split of this group. The presence of a ZZ/ZW system in $H$. suspectum (Johnson Pokorná et al. 2014) suggests that these sex chromosomes might be ancestral for Anguimorpha. A test of homology of varanid and helodermatid ZZ/ZW sex chromosomes needs to be performed in future studies to clarify this point.

The high conservation of sex chromosomes described here in monitor lizards has also been reported for iguanas (XY, Rovatsos et al. 2014b, 2014c; Altmanová et al. 2016, 2018), caenophidian snakes (ZW, Matsubara et al. 2006; Vicoso et al. 2013; Rovatsos et al. 2015) and lacertid lizards (ZW, Rovatsos et al. 2016). This stability is comparable to viviparous mammals (XY, Graves 2006; Waters et al. 2007) and birds (ZW, Shetty et al. 1999; Zhou et al. 2014).

The $\mathrm{W}$ chromosome in V. beccarii, V. macraei, V. prasinus, $V$. mertensi, $V$. glauerti, and $V$. acanthurus analyzed in this study was strongly heterochromatic as detected via C-banding (Fig. 2d, g, i, n, q, s). No heterochromatic microchromosome was detected in the female of $V$. salvator (Fig. 21). A sex ratio consistent with temperature-dependent sex determination was reported in this species by Hairston and Burchfield (1992). However, our chromosome painting results revealed ZZ/ZW sex chromosomes with extensive differences in genetic content between $\mathrm{Z}$ and $\mathrm{W}$ in $\mathrm{V}$. salvator. Therefore, heterochromatinization may not be a universal feature of differentiated sex chromosomes. The lack of heterochromatin detectable by $\mathrm{C}$-banding in $V$. salvator can be attributed to extensive variability in noncoding DNA in the $\mathrm{W}$ sex chromosomes of varanids. Unpaired chromosomes ( $\mathrm{Y}$ and $\mathrm{W}$ ) of vertebrates often undergo heterochromatinization and accumulation of repetitive sequences during sex chromosome differentiation (e.g., Acosta et al. 2009; Pokorná et al. 2011b, 2014; Matsubara et al. 2016; Augstenová et al. 2018). Evidence for a likely secondary elimination of a heterochromatic block on differentiated sex chromosomes has also been reported, for instance, in oplurid iguanas (Altmanová et al. 2016). The nested phylogenetic position of $V$. salvator suggests that secondary loss of heterochromatin from $\mathrm{W}$ chromosome might be plausible also for this species.

Until now, the dynamics of the genetic content of the W chromosome among varanids has only been partially investigated. Matsubara et al. (2014) performed a test among $V$. gouldii, $V$. rosenbergii, and $V$. acanthurus by hybridization of microsatellite motifs and the $\mathrm{W}$ chromosome probe obtained from $V$. acanthurus. Most sequences were apparently not conserved between $\mathrm{W}$ chromosomes because the chromosome $\mathrm{W}$ probe from $V$. acanthurus showed only weak hybridization signals on the pericentromeric region of the $\mathrm{W}$ chromosomes in $V$. rosenbergi and $V$. gouldii females. FISH with microsatellite motifs also revealed a different repetitive sequence composition between the $\mathrm{W}$ chromosomes of the three species. Johnson Pokorná et al. (2016) obtained similar results. These authors did not find any accumulation of the microsatellites accumulated in the $\mathrm{W}$ chromosome of $V$. acanthurus on the $\mathrm{W}$ chromosome of $V$. komodoensis. However, in our case, hybridization results of VKO11/12/W onto metaphases of all female individuals revealed a strong conservation of the W chromosomes among varanids. This discrepancy could be explained by methodological differences. The part of the W labeled by the probe derived from flow sorting can be rich in euchromatic regions containing coding genes, which are more conserved across species than repetitive sequences such as microsatellites diverging rapidly during evolution (Matsubara et al. 2006; Pokorná et al. 2011b; Rutkowska et al. 2012; Altmanová et al. 2016). These dynamics would explain size differences between $\mathrm{W}$ chromosomes among varanids despite sex chromosome homology.

\section{Triploidy in V. albigularis}

The fully grown individual of $V$. albigularis was found to have a chromosome number of $3 n=60$. King and King (1975) described the typical varanid chromosome number of $2 n=40$ for this species (referred there as $V$. exanthematicus albigularis). They also reported ZZ/ZW sex chromosomes for this species. Based on the results of the chromosome painting it seems that our individual possesses a ZZZ sex chromosome constitution. Although further investigations are still needed to clarify the origin of triploidy in $V$. albigularis, we tentatively assume that it emerged spontaneously in this individual. Spontaneous 
triploidy has been reported in sauropsids, including birds, iguanas, and snakes (Tiersch and Figiel 1991; Lamborot and Vásquez 1998 and citations therein; Stenberg and Saura 2013; Rovatsos et al. 2018). In amniotes it mostly leads to early mortality (Bonaminio and Fechheimer 1993; Baumer et al. 2000; Trukhina and Smirnov 2014). Triploidy is mainly attributed to dispermic fertilization of an egg or abnormal meiosis. However, in squamates it can be also the result of mating between a female of a diploid obligatory asexual hybrid and a male derived from one of the sexual ancestors (Moritz et al. 1989; Neaves and Baumann 2011; Trifonov et al. 2015; Ryskov et al. 2017). Facultative parthenogenetic births have been documented for several monitor lizards, such as $V$. panoptes, $V$. komodoensis, $V$. ornatus, V. glauerti, and V. rainerguentheri (Watts et al. 2006; Hennessy 2010; Wiechmann 2011, 2012; Hörenberg 2013; Grabbe and Koch 2014). The triploidy status of our individual $V$. albigularis albigularis could be the result of a parthenogenetic diploid egg followed by fertilization. Several parthenogenetic male individuals of $V$. komodoensis have been karyotyped by Johnson Pokorná et al. (2016) and all of them were diploids, however, the mother of these males did not have access to males at the time of clutch formation.

In conclusion, our study shows that genome organisation in monitor lizards is generally well conserved. Interchromosomal rearrangements were not observed, but we uncovered intrachromosomal rearrangements in four pairs of macrochromosomes during the evolution of the group. Our ancestral state reconstruction suggests that intrachromosomal rearrangements led to transitions from acrocentric to metacentric chromosomes and vice versa. We also documented the first case of triploidy among varanids. Sex chromosomes are clearly conserved across varanids during their long evolutionary history. Although homologous, the $\mathrm{W}$ chromosomes differ among varanid species in size, morphology, content of DNA repeats, and heterochromatinization. Future studies exploring and expanding our findings should investigate karyotype evolution in varanids at all molecular genomic levels. Sequencing and chromosome anchoring of V. komodoensis genome in the ongoing project will provide an important base for further research particularly in varanids and their anguimorphan outgroups where partial cytogenetic maps or genomic data are already available (Srikulnath et al. 2013; Gao et al. 2017).

Acknowledgements We are very grateful to our friends and colleagues who provided samples of monitor lizards for this study, among others, Tomáš Jirásek from Zoo Plzeň, Martina Gregorovičová, Nikola Ehlová, Lukáš Kubička, Barbora Augstenová, and Pavel Němec. The study was supported by Charles University PRIMUS Research Program (PRIMUS/SCI/46), Czech Science Foundation (17-22141Y), and Charles University Research Centre program (204069). A.I. received a $\mathrm{PhD}$ scholarship from the University of Florence, Italy.

\section{Compliance with ethical standards}

Conflict of interest The authors declare that they have no conflict of interest.

Publisher's note: Springer Nature remains neutral with regard to jurisdictional claims in published maps and institutional affiliations.

\section{References}

Acosta MJ, Marchal JA, Mitsainas GP, Rovatsos MT, FernándezEspartero CH, Giagia-Athanasopoulou EB et al. (2009) A new pericentromeric repeated DNA sequence in Microtus thomasi. Cytogenet Genome Res 124:27-36

Alföldi J, Di Palma F, Grabherr M, Williams C, Kong L, Mauceli E et al. (2011) The genome of the green anole lizard and a comparative analysis with birds and mammals. Nature 477:587-591

Altmanová M, Rovatsos M, Johnson Pokorná M, Veselý M, Wagner F, Kratochvíl L (2018) All iguana families with the exception of basilisks share sex chromosomes. Zoology 126:98-102

Altmanová M, Rovatsos M, Kratochvíl L, Johnson Pokorná M (2016) Minute $\mathrm{Y}$ chromosomes and karyotype evolution in Madagascan iguanas (Squamata: Iguania: Opluridae). Biol J Linn Soc 118:618-633

Augstenová B, Mazzoleni S, Kratochvíl L, Rovatsos M (2018) Evolutionary dynamics of the $\mathrm{W}$ chromosome in caenophidian snakes. Genes 9:5

Baumer A, Balmer D, Binkert F, Schinzel A (2000) Parental origin and mechanisms of formation of triploidy: a study of 25 cases. Eur J Hum Genet 8:911-917

Bonaminio G, Fechheimer N (1993) The gonadal histology of triploid chicken (Gallus domesticus) embryos. Genet Sel Evol 25:205-210

Chaiprasertsri N, Uno Y, Peyachoknagul S, Prakhongcheep O, Baicharoen S, Charernsuk S et al. (2013) Highly species-specific centromeric repetitive DNA sequences in lizards: Molecular cytogenetic characterization of a novel family of satellite DNA sequences isolated from the water monitor lizard (Varanus salvator macromaculatus, Platynota). J Hered 104:798-806

Dobigny G, Ducroz JF, Robinson TJ, Volobouev V (2004) Cytogenetics and cladistics. Syst Biol 53:470-484

Dutt K (1968) A karyotype study of Varanus monitor Linn. Caryologia 21:1-10

De Smet WHO (1981) Description of the orsein stained karyotypes of 136 lizard species (Lacertilia, Reptilia) belonging to the families Teiidae, Scincidae, Lacertidae, Cordylidae and Varanidae (Autarchoglossa). Acta Zool Pathol Antverp 76:407-420

Ferguson-Smith MA, Trifonov V (2007) Mammalian karyotype evolution. Nat Rev Genet 8:950-962

Fitch WM (1971) Toward defining the course of evolution: minimum change for a specified tree topology. Syst Zool 20:406-416

Gao J, Li Q, Wang Z, Zhou Y, Martelli P, Li F et al. (2017) Sequencing, de novo assembling, and annotating the genome of the endangered Chinese crocodile lizard Shinisaurus crocodilurus. Gigascience 6:1-6

Giovannotti M, Caputo V, O’Brien PCM, Lovell FL, Trifonov V, Cerioni PN et al. (2010) Skinks (Reptilia: Scincidae) have highly conserved karyotypes as revealed by chromosome painting Cytogenet Genome Res 127:224-231

Gorman GC, Gress F (1970) Chromosome cytology of four boid snakes and a varanid lizard, with comments on the cytosystematics of primitive snakes. Herpetologica 26:308-317

Grabbe J, Koch A (2014) First and repeated cases of parthenogenesis in the varanid subgenus Euprepiosaurus (Varanus indicus species 
group) and the first successful breeding of $V$. rainerguentheri in captivity. Biawak 8:79-87

Graves JAM (2006) Sex chromosome specialization and degeneration in mammals. Cell 124:901-914

Hairston CS, Burchfield PM (1992) The reproduction and husbandry of the water monitor Varanus salvator at the Gladys Porter Zoo, Brownsville. Int Zoo Yearb 31:124-130

Hennessy J (2010) Parthenogenesis in an ornate Nile monitor, Varanus ornatus. Biawak 4:26-30

Hörenberg T (2013) Parthenogenese bei Varanus glauerti. Draco 53:29-30

Iannucci A, Altmanová M, Ciofi C, Ferguson-Smith M, Pereira JC, Rehák I et al. (2019). Isolating chromosomes of the Komodo dragon: new tools for comparative mapping and sequence assembly. Cytogenet Genome Res, in press, https://doi.org/10. $1159 / 000496171$

Janzen FJ, Krenz JG (2004) Phylogenetics: which was first, TSD or GSD? In: Valenzuela N, Lance VA (eds). Temperature dependent sex determination in vertebrates. Smithsonian Books, Washington, $\mathrm{p}$ 121-130

Johnson Pokorná M, Altmanová M, Rovatsos M, Velenský P, Vodička R, Rehák I et al. (2016) First description of the karyotype and sex chromosomes in the Komodo dragon (Varanus komodoensis). Cytogenet Genome Res 148:284-291

Johnson Pokorná M, Kratochvíl L (2016) What was the ancestral sexdetermining mechanism in amniote vertebrates? Biol Rev 91:1-12

Johnson Pokorná M, Rovatsos M, Kratochvíl L (2014) Sex chromosomes and karyotype of the (nearly) mythical creature, the Gila monster, Heloderma suspectum (Squamata: Helodermatidae). PLoS ONE 9:e104716

Johnson Pokorná M, Trifonov VA, Rens W, Ferguson-Smith MA, Kratochvíl L (2015) Low rate of interchromosomal rearrangements during old radiation of gekkotan lizards (Squamata: Gekkota). Chromosome Res 23:299-309

King M, King D (1975) Chromosomal evolution in the lizard genus Varanus (Reptilia). Aust J Biol Sci 28:89-108

King M, Mengden GA, King D (1982) A pericentric-inversion polymorphism and a ZZ/ZW sex-chromosome system in Varanus acanthurus Boulenger analysed by G- and C-banding and Ag staining. Genetica 58:39-45

Lamborot M, Vásquez M (1998) A triploid lizard (Liolaemus gravenhorsti) from Chile. J Herpetol 32:617-620

Lin L-H, Wiens JJ (2017) Comparing macroecological patterns across continents: evolution of climatic niche breadth in varanid lizards. Ecography 40:960-970

Lithgow PE, O'Connor R, Smith D, Fonseka G, Al Mutery A, Rathje $\mathrm{C}$ et al. (2014) Novel tools for characterising inter and intra chromosomal rearrangements in avian microchromosomes. Chromosome Res 22:85-97

Maddison WP, Maddison DR (2018). Mesquite: a modular system for evolutionary analysis. Version $3.51 \mathrm{http} / / /$ mesquiteproject.org

Matsubara K, O’Meally D, Azad B, Georges A, Sarre SD, Graves JA et al. (2016) Amplification of microsatellite repeat motifs is associated with the evolutionary differentiation and heterochromatinization of sex chromosomes in Sauropsida. Chromosoma 125:111-123

Matsubara K, Sarre SD, Georges A, Matsuda Y, Graves JAM, Ezaz T (2014) Highly differentiated ZW sex microchromosomes in the Australian Varanus species evolved through rapid amplification of repetitive sequences. PLoS ONE 9:e95226

Matsubara K, Tarui H, Toriba M, Yamada K, Nishida-Umehara C, Agata K et al. (2006) Evidence for different origin of sex chromosomes in snakes, birds, and mammals and step-wise differentiation of snake sex chromosomes. Proc Natl Acad Sci 103:18190-18195
Matthey R (1931) Chromosomes de sauriens: Helodermatidae, Varanidae, Xantusiidae, Anniellidae, Anguidae. Bull Soc Vaud Sci Nat 57:269-270

Moritz C, Donnellan S, Adams M, Baverstock PR (1989) The origin and evolution of parthenogenesis in Heteronotia binoei (Gekkonidae): extensive genotypic diversity among parthenogens. Evolution 43:994-1003

Neaves WB, Baumann P (2011) Unisexual reproduction among vertebrates. Trends Genet 27:81-88

Olmo E, Signorino GG (2016). Chromorep: a reptiles chromosomes database. http://chromorep.univpm.it/ Accessed 16 October 2017

Patawang I, Tanomtong A (2017a) Constitutive heterochromatin observed on metaphase chromosome of Varanus bengalensis by C-banding and DAPI methods. Cytol (Tokyo) 82:1

Patawang I, Tanomtong A, Getlekha N, Phimphan S, Pinthong K, Neeratanaphan L (2017b) Standardized karyotype and idiogram of bengal monitor lizard, Varanus bengalensis (Squamata, Varanidae). Cytol (Tokyo) 82:75-82

Pianka ER, King DR (2004) Varanoid lizards of the world. Indiana University Press, Bloomington

Pokorná M, Giovannotti M, Kratochvíl L et al. (2011a) Strong conservation of the bird $\mathrm{Z}$ chromosome in reptilian genomes is revealed by comparative painting despite 275 million years divergence. Chromosoma 120:455-468

Pokorná M, Giovannotti M, Kratochvíl L, Caputo V, Olmo E, Ferguson-Smith MA et al. (2012) Conservation of chromosomes syntenic with avian autosomes in squamate reptiles revealed by comparative chromosome painting. Chromosoma 121:409-418

Pokorná M, Kratochvíl L (2009) Phylogeny of sex-determining mechanisms in squamate reptiles: are sex chromosomes an evolutionary trap? Zool J Linn Soc 156:168-183

Pokorná M, Kratochvíl L, Kejnovský E (2011b) Microsatellite distribution on sex chromosomes at different stages of heteromorphism and heterochromatinization in two lizard species (Squamata: Eublepharidae: Coleonyx elegans and Lacertidae: Eremias velox). BMC Genet 12:90

Pokorná M, Rábová M, Ráb P, Ferguson-Smith MA, Rens W, Kratochvíl L (2010) Differentiation of sex chromosomes and karyotypic evolution in the eye-lid geckos (Squamata: Gekkota: Eublepharidae), a group with different modes of sex determination. Chromosome Res 18:809-820

Pokorná M, Rens W, Rovatsos M, Kratochvíl L (2014) A ZZ/ZW sex chromosome system in the thick-tailed gecko (Underwoodisaurus milii; Squamata: Gekkota: Carphodactylidae), a member of the ancient gecko lineage. Cytogenet Genome Res 142:190-196

Porter C, Haiduk M, De Queiroz K (1994) Evolution and phylogenetic significance of ribosomal gene location in chromosomes of squamate reptiles. Copeia 1994:302-313

Pyron RA, Burbrink FT, Wiens JJ (2013) A phylogeny and revised classification of Squamata, including 4161 species of lizards and snakes. BMC Evol Biol 13:93

Rovatsos M, Altmanová M, Johnson Pokorná M, Velenský P, Sánchez Baca A, Kratochvíl L (2017) Evolution of karyotypes in chameleons. Genes 8:382

Rovatsos M, Altmanová M, Johnson Pokorná M, Kratochvíl L (2014a) Novel X-linked genes revealed by quantitative polymerase chain reaction in the green anole, Anolis carolinensis. G3 4:2107-2113

Rovatsos M, Altmanová M, Pokorná M, Kratochvíl L (2014b) Conserved sex chromosomes across adaptively radiated Anolis lizards. Evolution 68:2079-2085

Rovatsos M, Augstenová B, Altmanová M, Sloboda M, Kodym P, Kratochvíl L (2018) Triploid colubrid snake provides insight into the mechanism of sex determination in advanced snakes. Sex Dev $12: 251-255$ 
Rovatsos M, Pokorná M, Altmanová M, Kratochvíl L (2014c) Cretaceous park of sex determination: sex chromosomes are conserved across iguanas. Biol Lett 10:20131093

Rovatsos M, Vukić J, Altmanová M, Johnson Pokorná M, Moravec J, Kratochvíl L (2016) Conservation of sex chromosomes in lacertid lizards. Mol Ecol 25:3120-3126

Rovatsos M, Vukić J, Lymberakis P, Kratochvíl L (2015) Evolutionary stability of sex chromosomes in snakes. Proc R Soc B Biol Sci 282:20151992

Rutkowska J, Lagisz M, Nakagawa S (2012) The long and the short of avian $\mathrm{W}$ chromosomes: No evidence for gradual $\mathrm{W}$ shortening. Biol Lett 8:636-638

Ryskov AP, Osipov FA, Omelchenko AV, Semyenova SK, Girnyk AE, Korchagin VI et al. (2017) The origin of multiple clones in the parthenogenetic lizard species Darevskia rostombekowi. PLoS ONE 12:e0185161

Sarre SD, Georges A, Quinn A (2004) The ends of a continuum: genetic and temperature-dependent sex determination in reptiles. Bioessays 26:639-645

Shetty S, Griffin DK, Graves JAM (1999) Comparative painting reveals strong chromosome homology over 80 million years of bird evolution. Chromosome Res 7:289-295

Shibaike Y, Takahashi Y, Arikura I, Iiizumi R, Kitakawa S, Sakai M et al. (2009) Chromosome evolution in the lizard genus Gekko (Gekkonidae, Squamata, Reptilia) in the East Asian islands. Cytogenet Genome Res 127:182-190

Singh L (1972) Evolution of karyotypes in snakes. Chromosoma 38:185-236

Singh L (1974) Study of mitotic and meiotic chromosomes in seven species of lizards. Proc Zool Soc 27:57-79

Singh L, Sharma T, Ray-Chaudhu SP (1970) Chromosome numbers and sex chromosomes in few Indian species of amphibia and reptiles. Mamm Chrom News 11:91-94

Skinner BM, Griffin DK (2012) Intrachromosomal rearrangements in avian genome evolution: evidence for regions prone to breakpoints. Heredity 108:37-41

Srikulnath K, Uno Y, Nishida C, Matsuda Y (2013) Karyotype evolution in monitor lizards: cross-species chromosome mapping of cDNA reveals highly conserved synteny and gene order in the Toxicofera clade. Chromosome Res 21:805-819

Stenberg P, Saura A (2013) Meiosis and its deviations in polyploid animals. Cytogenet Genome Res 140:185-203

Sumner AT (1972) A simple technique for demonstrating centromeric heterochromatin. Exp Cell Res 75:304-306

Tiersch TR, Figiel CR (1991) A triploid snake. Copeia 1991:838-841

Trifonov VA, Giovannotti M, O'Brien PCM, Wallduck M, Lovell F, Rens W et al. (2011) Chromosomal evolution in Gekkonidae. I.
Chromosome painting between Gekko and Hemidactylus species reveals phylogenetic relationships within the group. Chromosome Res 19:843-855

Trifonov VA, Paoletti A, Caputo Barucchi V, Kalinina T, O’Brien PC, Ferguson-Smith MA, Giovannotti M (2015) Comparative chromosome painting and NOR distribution suggest a complex hybrid origin of triploid Lepidodactylus lugubris (Gekkonidae). PLoS ONE 10:1-13

Trukhina AV, Smirnov AF (2014) Problems of birds sex determination. Nat Sci 6:1232-1240

Uetz P, Hošek J (2017). The reptile database. http://www.reptile-data base.org/. Accessed 16 October 2017

Valenzuela N, Adams DC, Janzen FJ (2003) Pattern does not equal process: exactly when is sex environmentally determined? Am Nat 161:676-683

Valenzuela N, Lance VA (2004) Temperature dependent sex determination in vertebrates. Smithsonian Books, Washington

Vicoso B, Emerson JJ, Zektser Y, Mahajan S, Bachtrog D (2013) Comparative sex chromosome genomics in snakes: differentiation, evolutionary strata, and lack of global dosage compensation. PLoS Biol 11:e1001643

Vidal N, Hedges SB (2005) The phylogeny of squamate reptiles (lizards, snakes, and amphisbaenians) inferred from nine nuclear protein-coding genes. Comptes Rendus - Biol 328:1000-1008

Vidal N, Marin J, Sassi J, Battistuzzi FU, Donnellan S, Fitch AJ et al. (2012) Molecular evidence for an Asian origin of monitor lizards followed by Tertiary dispersals to Africa and Australasia. Biol Lett 8:853-855

Waters PD, Wallis MC, Marshall Graves JA (2007) Mammalian sexorigin and evolution of the Y chromosome and SRY. Semin Cell Dev Biol 18:389-400

Watts PC, Buley KR, Sanderson S, Boardman W, Ciofi C, Gibson R (2006) Parthenogenesis in Komodo dragons. Nature 444:1021-1022

Wiechmann R (2011) Eigene Beobachtungen zur Parthenogenese bei Waranen. Elaphe 19:55-61

Wiechmann R (2012) Observations on parthenogenesis in monitor lizards. Biawak 6:11-21

Zheng Y, Wiens JJ (2016) Combining phylogenomic and supermatrix approaches, and a time-calibrated phylogeny for squamate reptiles (lizards and snakes) based on 52 genes and 4162 species. Mol Phylogenet Evol 94:537-547

Zhou Q, Zhang J, Bachtrog D, An N, Huang Q, Jarvis ED et al. (2014) Complex evolutionary trajectories of sex chromosomes across bird taxa. Science 346:1246338 